\title{
Strategic Games in Groundwater Management
}

\author{
Catarina ROSETA-PALMA Ana Brasão
}

\begin{abstract}
Previous work has shown that quality-quantity interactions may alter the typical results of private management under the assumption of myopic behavior. The main objective of the paper is to analyse the role of feedback strategies in a model containing an integrated quantity-quality approach, analyzing the impact on water use, pollution and shadow resource prices.

The case of symmetric players is shown to yield results that are similar to those of models in previous literature, in that it establishes myopic and optimal solutions as extremes, with feedback solutions somewhere in between. However, as different water users do not have similar objectives or constraints, it is important to consider the case of asymmetric players. This paper shows that when agents are asymmetric, especially as regards external effects, strategic solutions can be more extreme than the myopic ones.
\end{abstract}

Keywords: feedback Nash strategies, common property externalities, groundwater management, asymmetric players 


\section{Introduction ${ }^{1}$}

Groundwater is often exploited as a common property resource, with access granted to a number of agents who pump water according to their own interests. Moreover, some water uses can produce significant changes in water quality with negative implications for other agents. Both quantity and quality externalities are widespread, so it would be natural to suppose that optimal management brings about a larger aquifer of better quality than what noncooperating agents would generate in a common property setting. It has been shown that this is not necessarily true: intervention may lower quantity while improving quality, or even lower quality while improving quantity (the exact result will depend, among other things, on the properties of the water quality regeneration function. See Roseta-Palma (2002)) However, those results were obtained considering that agents in a common property setting behaved myopically, ignoring both the dynamics of the aquifer and the actions of other users. The use of the myopic assumption as representative of "competitive" (ie. unregulated) common property management in groundwater goes back at least to the seminal paper by Gisser and Sanchez (1980). Yet the natural characteristics of groundwater indicate that users may take less shortsighted decisions. This paper develops a model of joint quality-quantity management in a dynamic game framework, considering both the stock of water quantity and a stock measure of water quality and analysing different assumptions of behaviour for the water users. In particular, the myopic and the feedback Nash solutions are examined and compared to the optimal solution.

The myopic solution is obtained considering that agents are essentially oblivious to the dynamics of interaction between players and to the movements of state variables. Thus each agent selects his control variables (water extraction as well as possible contaminant use) to maximize individual current profit. In reality, access to groundwater is usually restricted to a well known area where there are a fixed number of wells, so models of strategic behaviour seem to provide a more appropriate setting. In the context of an n-player noncooperative diferential game,

\footnotetext{
${ }^{1}$ Support from Fundação para a Ciência e Tecnologia, project POCTI/ECO/41127/2001 is gratefully acknowledged.
} 
open-loop Nash equilibria consist of solutions where each player makes his own decisions while taking the specific decisions of other players are as given. This type of solution requires each player to choose from the outset the entire temporal paths for control variables that it will commit to, thus it is not very realistic either. The analysis of feedback Nash equilibria, on the other hand, is carried out considering that each player chooses strategies consisting of rules for the control variable. These feedback strategies are rules that result in actions of the players conditional on the current values of state variables and, therefore, require agents to adjust their decisions in each period according to the observed state of the system, but continue using their strategy independently of deviations by the other players.

Several papers have considered feedback strategies in environmental problems of pollution control (see for example Xepapadeas (1995), Dockner and Long (1993), List and Mason (2001)). The case of groundwater management in particular has been dealt with in Xepapadeas (1996), which considers a quantity-quality problem where contamination depends only on the amount of water pumped, in Provencher and Burt (1993), which analyses the impact of strategical behavior on the efficiency of the common property solution, and in Rubio and Casino (2001), which contains a quantity-only management model and investigates nonlinear feedback strategies. All of these papers consider symmetrical water users and most establish myopic and optimal solutions as extremes, with feedback solutions somewhere in between. The main objective of this paper is to extend the feedback common property framework to a model containing an integrated quantity-quality approach, analyzing the impact on water use, pollution and shadow resource prices. Moreover, as different economic agents do not have similar objectives or constraints, it is important to consider the case of asymmetric players. In particular, the paper develops a model that distinguishes between agricultural use and public water supply, and analyzes the resulting interactions under alternative common property arrangements. The main result is that the strategic considerations inherent to the feedback solution may take agents even further away from the optimum than what be expected if common property management was myopic. The symmetric case is presented in the following section, 
for comparison with previous results in the literature and also to highlight the novelty of the asymmetric model results.

\section{Symmetric model}

In this section it is assumed that $M$ identical agents exploit a stock of groundwater that receives a constant recharge, $R$. The size of the aquifer is reflected on the pumping lift, $L$, defined as the distance between the land surface and the water level. Water quality is represented by the level of contamination, $C$, which affects the profitability of water use. All agents produce some good using water and another input according to a production function $y\left(g_{t}, n_{t} ; C_{t}\right)$, where $g_{t}$ is the amount of water used and $n_{t}$ is the amount of an input whose use creates pollution. It is are assumed that there are positive but diminishing marginal returns for both inputs: ${ }^{2} \frac{\partial u}{\partial g}, \frac{\partial y}{\partial n}>0, \frac{\partial^{2} u}{\partial g^{2}}, \frac{\partial^{2} u}{\partial n^{2}}<0$ and that they are complementary in production $\frac{\partial^{2} y}{\partial g \partial n}>0$. The influence of water contamination on production could take different forms (see Roseta-Palma (2002)), but the most commonly used assumptions denote the idea that contaminated water reduces both total production and the marginal productivities of inputs: $\frac{\partial y}{\partial C}<0, \frac{\partial^{2} y}{\partial g \partial C}<0$, and $\frac{\partial^{2} y}{\partial n \partial C}<0$.

The cost of groundwater extraction depends on lift and on the amount of water pumped, $k\left(g_{t}, L_{t}\right)$. In this case pumping costs are assumed to take the form of a constant unit cost $k$ per unit of water pumped per distance lifted, ie. $k g_{t} L_{t}$. Thus the current net benefit of production at $t$ for each agent is given by

$$
\pi_{t}=P_{y} y\left(n_{t}, g_{t} ; C_{t}\right)-k\left(g_{t}, L_{t}\right)-P_{n} n_{t}
$$

where $P_{y}$ is the price of $y$ and $P_{n}$ is the price of $n$. The evolution of lift, $L$ can be represented by:

$$
\dot{L}=\frac{(1-\alpha) g^{T}-R}{A S}
$$

where $g^{T}=M g_{t}$ is the total water used, $\alpha$ is the return coefficient (which can be zero for fully consumptive uses), $A$ is aquifer area and $S$ is the specific yield. It

\footnotetext{
${ }^{2}$ The $t$ subscript is dropped to simplify notation whenever possible.
} 
is assumed that there is a constant natural decay rate for the pollutant, so that contamination evolves according to:

$$
\dot{C}=M e\left(n_{t}\right)-\delta C_{t}
$$

Thus water quality degrades as it receives contaminant loads, which originate on the surface and percolate towards groundwater according to a pollution production function that depends positively on the amount of polluting input. ${ }^{3}$. To preserve symmetry between agents, the evolution of both contamination and lift is assumed to affect all users homogeneously and instantaneously.

If the behavior of the agents is myopic, decisions on inputs are taken to maximize (1), which for interior solutions implies first order conditions:

$$
\begin{aligned}
& P_{y} \frac{\partial y}{\partial g}=k L_{t} \\
& P_{y} \frac{\partial y}{\partial n}=P_{n}
\end{aligned}
$$

This solution equates marginal benefit of inputs to the individual agent with their marginal costs, and is inefficient since it ignores effects on the state variables as well as on other agents. Given a discount rate $\rho$, the optimal management solution requires $\max M \int_{0}^{\infty} \pi_{t} e^{-\rho t} d t$ subject to (2) and (3), as well as initial conditions for $L$ and $C$ and non-negativity restrictions on inputs and $C$. In general, for unconfined aquifers $L$ will also be non-negative and it will be further restricted by some maximum value. Using the current value Hamiltonean

$\mathcal{H}=M\left[P_{y} y\left(n_{t}, g_{t} ; C_{t}\right)-k g_{t} L_{t}-P_{n} n_{t}\right]+\lambda_{t}\left[\frac{(1-\alpha) M g_{t}-R}{A S}\right]+\beta_{t}\left[M e\left(n_{t}\right)-\delta C_{t}\right]$

\footnotetext{
${ }^{3}$ In general, the contaminant loads could depend on $g_{t}$ as well as $n_{t}$. However, the role of applied water is ambiguous, since it might act as a carrier for pollutants or diminish the load, either by dilution or by promoting plant uptake (in the case of agriculture).
} 
optimal conditions for interior solutions are:

$$
\begin{aligned}
P_{y} \frac{\partial y}{\partial g} & =k L_{t}-\lambda_{t} \frac{(1-\alpha)}{A S} \\
P_{y} \frac{\partial y}{\partial n} & =P_{n}-\beta_{t} \frac{\partial e}{\partial n} \\
\dot{\lambda} & =\rho \lambda_{t}+k g_{t} M \\
\dot{\beta} & =(\rho+\delta) \beta_{t}-P_{y} \frac{\partial y}{\partial C} M
\end{aligned}
$$

Comparing equations (4) and (5) with (6) and (7), with both co-state variables taking negative values (as they are the shadow prices for state variables that are detrimental in the objective function), there are additional terms on the optimal conditions. These represent the extra marginal costs of input use through their impact on state variables, and will ensure that for given values of $L$ and $C$ optimal input use will be lower than the individual myopic decision. However, even if initial conditions are similar, after different decisions are taken the paths for $L$ and $C$ will not coincide, and paths for $g$ and $n$ cannot be contrasted in general. Nonetheless, steady state conditions can be analysed and compared. Any steady state will be defined by $\dot{L}=0, \dot{C}=0$, yielding

$$
\begin{aligned}
g^{S S} & =\frac{R}{M(1-\alpha)} \\
C^{S S} & =\frac{M e\left(n^{S S}\right)}{\delta}
\end{aligned}
$$

Equation (10) indicates that the steady state amount of water that can be pumped by each agent is entirely defined by natural recharge and the number of agents, and it will not depend on the management arrangement. Equation (11) indicates the steady state relationship between contamination and input use, which is positive since $\frac{\partial e}{\partial n}>0$. Using the steady state conditions, it can be seen from equations (5) and (7) that contamination levels and input use will be higher in the private, myopic solution. However, from equations (4) and (6), optimal steady state lift may be higher or lower. In particular, $L_{o p t}^{S S}<L_{m b}^{S S}$ unless $\frac{\partial y}{\partial g_{o p t}}{ }_{\text {opt }}$ is sufficiently larger than $\frac{\partial y}{\partial g} S S$, in which case the opposite occurs. The possibility of 
a smaller water stock (higher lift) in the optimal case can be explained by recalling that the water is of higher quality and thus more productive.

Under the assumption of feedback strategies, each agent is expected to have a state-dependent decision rule. The simplest case is that of linear Markov strategies, which although it is rather crude can be a sensible assumption in certain circumstances, namely when the dynamic game has a linear quadratic structure. Rubio and Casino (2001) discuss the use of nonlinear strategies in a quantity-only groundwater management problem and conclude that, given restricted initial conditions (such as a natural hydrologic steady state), the linear strategy may perform better since it may be the only strategy that leads to a stable steady state. ${ }^{4}$ In the present case, with two control and two state variables, and considering the nature of the agent's problem, it will be assumed that the relevant linear strategies for each player $i$ consist, in each moment $t$, of

$$
\begin{aligned}
g_{t}^{i} & =\bar{g}^{i}-\gamma_{1} L_{t}-\gamma_{2} C_{t} \\
n_{t}^{i} & =\bar{n}^{i}-\nu_{1} L_{t}-\nu_{2} C_{t}
\end{aligned}
$$

where $\gamma_{1}, \gamma_{2}$ and $\nu_{1}, \nu_{2}$ are parameters representing the reaction of the decision variables to the values observed for the state variables. The same rules are applied by all the players, since they are assumed to be identical, and the reaction parameters are invariant with time.

In this setting, each agent solves problem $\max \int_{0}^{\infty} \pi_{t}^{i} e^{-\rho t} d t$ assuming that the

\footnotetext{
${ }^{4}$ Tsutsui and Mino (1990) examine the possibility of obtaining a more efficient equilibrium using nonlinear strategies in a duopoly with sticky prices, without guessing the type of value function. However, their method is applicable to the case of one state variable.
} 
laws of motion for lift and contamination are:

$$
\begin{aligned}
\dot{L} & =\frac{(1-\alpha)\left[g_{t}^{i}+\sum_{j \neq i}\left(\bar{g}^{j}-\gamma_{1} L_{t}-\gamma_{2} C_{t}\right)\right]-R}{A S} \\
& =\frac{(1-\alpha)\left[g_{t}^{i}+(M-1)\left(\bar{g}^{j}-\gamma_{1} L_{t}-\gamma_{2} C_{t}\right)\right]-R}{A S} \\
\dot{C} & =e\left(n_{t}^{i}\right)+\sum_{j \neq i} e\left(\bar{n}^{j}-\nu C_{t}\right)-\delta C_{t} \\
& =e\left(n_{t}^{i}\right)+(M-1) e\left(\bar{n}^{j}-\nu C_{t}\right)-\delta C_{t}
\end{aligned}
$$

The corresponding first order conditions for $g$ and $n$ are the same as in the optimal case, equations (6) and (7), except the shadow prices of $L$ and $C$ will take on different values, reflecting the individual perspective on the laws of motion. This is reflected in the evolution of shadow prices:

$$
\begin{aligned}
& \dot{\lambda}^{i}=\left(\rho+\frac{1-\alpha}{A S}(M-1) \gamma_{1}\right) \lambda_{t}^{i}+k g_{t}^{i}+\beta_{t}^{i}(M-1) \frac{\partial e}{\partial n} \nu_{1} \\
& \dot{\beta}^{i}=\left(\rho+\delta+\frac{\partial e}{\partial n}(M-1) \nu_{2}\right) \beta_{t}^{i}-P_{y} \frac{\partial y}{\partial C}+\frac{1-\alpha}{A S}(M-1) \gamma_{2} \lambda_{t}^{i}
\end{aligned}
$$

At the steady state, given symmetry, equations (10) and (11) hold, as well as

$$
\begin{aligned}
\lambda^{i S S} & =-\frac{k g^{S S}+\beta^{i S S}(M-1) \frac{\partial e}{\partial n} \nu_{1}}{\rho+\frac{1-\alpha}{A S}(M-1) \gamma_{1}} \\
\beta^{i S S} & =\frac{P_{y} \frac{\partial y}{\partial C}-\frac{1-\alpha}{A S}(M-1) \gamma_{2} \lambda^{i S S}}{\rho+\delta+\frac{\partial e}{\partial n}(M-1) \nu_{2}}
\end{aligned}
$$

Comparing these expressions with the equivalent ones from the optimal case (which derive from setting the movement equations (8) and (9) to zero), it can be concluded that both shadow prices used by the common property agents in a feedback setting are smaller in absolute value than the optimal ones. ${ }^{5}$ Thus, as long as $\lambda^{i S S}$ and $\beta^{i S S}$ remain negative, which they should since they are the same for all players and it would not make sense for lift or contamination to be

\footnotetext{
${ }^{5}$ The result is unambiguous provided $\left|\frac{\partial u}{\partial C}^{i S S}\right|<\left|\frac{\partial u}{\partial C}{ }_{o p t}^{S S} M\right|$.
} 
seen as "good" for everyone, first order conditions indicate that both steady state contamination and steady state lift will be between the myopic and the optimal values. This result is similar to other results in the literature (see for example Rubio and Casino (2001), Xepapadeas (1995)).

\section{Two distinct groups of agents}

In this section we distinguish between two types of players with different characteristics. In particular, we consider two water uses that give rise to different benefits but also have distinct quality requirements: farming and public supply. The particular problem that is studied is typical of agricultural areas where irrigation is significant and other water sources are not abundant, such as in many aquifers in the south of the Iberian Peninsula. On the one hand, there is a group of farmers who extract water from the underlying aquifer to irrigate their crops; not only does this reduce availability of water quantitatively, but the discharges from this group of players are also assumed to worsen the quality of the remaining groundwater. ${ }^{6}$ On the other hand, there are urban users, represented by a public water supplier who also retrieves water from the same aquifer. This second group of users has quality requirements, which give rise to treatment costs anytime the water is too contaminated. In this model, although quantity externalities arise from both users' decisions, the contamination externality is only from the farmers to the urban users. There could also be "within group" externalities, especially among farmers, but those are assumed to be comparatively unimportant so that each group can be treated as a single agent.

The problem for the group of agricultural users is summarized by a net benefit function in each period:

$$
\pi_{a}=P_{y} y\left(n, g_{a}\right)-k g_{a} L-P_{n} n
$$

where $g_{a}$ is the amount of groundwater pumped by this group and other variables are as before ( $n$ represents a polluting input used in agricultural production, such

\footnotetext{
${ }^{6}$ These quality worsening discharges are not necessarily associated with the use of polluting inputs. For instance, salinity in aquifers can be caused directly by water withdrawals.
} 
as fertiliser.or pesticides).

As for public supply, the only decision variable is how much water to take from the aquifer for domestic users $\left(g_{d}\right)$. Given a negatively sloped water demand function, $P_{d}(g)$, with the associated consumer surplus, and given treatment costs $k_{T}\left(C, g_{d}\right)=k_{T} g_{d} C$, net benefit can be expressed as:

$$
\pi_{d}=\int_{0}^{g_{d}} P_{d}(g) d g-k g_{d} L-k_{T} g_{d} C
$$

The optimal solution to this management problem involves the maximization of $\int_{0}^{\infty}\left(\pi_{a}+\pi_{d}\right) e^{-\rho t} d t$ on the three decision variables, subject to the movement equations (2) and (3), duly adapted to reflect the structure of the asymetric problem, as well as initial conditions and relevant restrictions. In particular, equation (2) will now represent total water extraction as the sum of both users' withdrawals, corrected by the return coefficient for $g_{a}$ but not for $g_{d}$, which normally will go into a sewage system and not percolate back down to the aquifer:

$$
\dot{L}=\frac{(1-\alpha) g_{a}+g_{d}-R}{A S}
$$

First order conditions can be summarized as:

$$
\begin{aligned}
P_{y} \frac{\partial y}{\partial g_{a}} & =k L-\lambda \frac{(1-\alpha)}{A S} \\
P_{d}\left(g_{d}\right) & =k L+k_{T} C-\frac{\lambda}{A S} \\
P_{y} \frac{\partial y}{\partial n} & =P_{n}-\beta \frac{\partial e}{\partial n} \\
\dot{\lambda} & =\rho \lambda+k\left(g_{a}+g_{d}\right) \\
\dot{\beta} & =(\rho+\delta) \beta+k_{T} g_{d}
\end{aligned}
$$

Conditions (25) and (27) are similar to the previous case. Condition (26) equates marginal costs and benefits of water extraction for domestic users. Note that the shadow price of lift, $\lambda$, appears in both (25) and (26), and its path is explained by the impact of lift on pumping costs (equation 28). Finally, equation 
(29) shows the evolution of the shadow price of contamination, reflecting only its effect on treatment costs of domestic users since by assumption there is no impact on agricultural productivity. Both shadow prices are negative at the optimal steady state.

To describe the myopic solution, the first three equations above can be used, simply setting $\lambda$ and $\beta$ to zero. Some comparisons can be established between the myopic and the optimal case, but different combinations are possible. Initially, myopic users of both types will pump more than they should and farmers will apply more of the polluting input, so that the state variables of the system will diverge. At the steady state, the picture is not as clear as in the symmetric case, because $\dot{L}=0$ now yields $(1-\alpha) g_{a}^{S S}+g_{d}^{S S}=R$, implying that different management schemes can result in different ways of sharing the natural recharge among the two groups. Instead of highligthing the possible comparisons that can be made between these two steady state solutions, we will proceed henceforth to the feedback equilibrium, which yields more interesting results.

The agricultural group will decide $g_{a}$ and $n$ assuming a strategy for $g_{d}$ based on $L$ and $C$, since both of these variables affect public supply costs, $g_{d}=g_{d}(L, C)$. The domestic side, on the other hand, will select $g_{d}$ assuming that strategies for both $g_{a}$ and $n$ depend only on $L, g_{a}(L)$ and $n(L) .{ }^{7}$ The equilibrium (or equilibria,

\footnotetext{
${ }^{7}$ Recall that feedback strategies are based on each user taking the other's strategy as given, so that perceived decisions for the other at any time are based only on current values of state variables. No one takes into consideration that those values in turn depend on their own decisions. If one user did acknowledge that indirect effect of his own decisions, it would be a Stackelberg game setting. However, in many continuous time models the two types of games coincide (see Rubio (2003)).
} 
in general) will be given by the simultaneous solution of the following two systems:

$$
\begin{aligned}
P_{y} \frac{\partial y}{\partial g_{a}} & =k L-\lambda_{a} \frac{(1-\alpha)}{A S} \\
P_{y} \frac{\partial y}{\partial n} & =P_{n}-\beta_{a} \frac{\partial e}{\partial n} \\
\dot{\lambda}_{a} & =\left(\rho-\frac{\frac{\partial g_{d}}{\partial L}}{A S}\right) \lambda_{a}+k g_{a} \\
\dot{\beta}_{a} & =(\rho+\delta) \beta_{a}-\frac{\frac{\partial g_{d}}{\partial C}}{A S} \lambda_{a}
\end{aligned}
$$

and

$$
\begin{aligned}
P_{d}\left(g_{d}\right) & =k L+k_{T} C-\frac{\lambda_{d}}{A S} \\
\dot{\lambda}_{d} & =\left(\rho-\frac{(1-\alpha) \frac{d g a}{d L}}{A S}\right) \lambda_{d}+k g_{d}-\beta_{d} \frac{d e}{d n} \frac{d n}{d L} \\
\dot{\beta}_{d} & =(\rho+\delta) \beta_{d}+k_{T} g_{d}
\end{aligned}
$$

One immediately noticeable thing about this solution is that shadow prices will be distinct for $a$ and $d$. In fact, it can easily be seen from equations (33) and (36) that at the steady state $\beta_{a}$ and $\beta_{d}$ actually have opposite signs. The agricultural group, which is not directly affected by contamination, will strategically consider lower water quality as a good thing, because it reduces the quantity of water extracted for public supply. A similar disparity may arise on $\lambda$, but in this case shadow prices do not always have opposite signs as each user weighs his own direct costs of increasing lift against the indirect benefits from decreasing the other user's withdrawals. Hence, although it is difficult to get general results for steady state $L$ and $C$ for each of the three situations (myopic, feedback, and optimal), comparing the shadow prices and the resulting marginal net benefits for each decision variable, a new result appears, which is that the strategic considerations inherent to the feedback solution may take agents even further away from the optimum than what would be expected if common property management was myopic. Such a result arises from the explicit consideration of asymmetric 
characteristics and carries some policy implications. For instance, if taxes were to be introduced in order to correct existing externalities, the analysis of agents' behavior regarding each other is of utmost importance. The myopic solution is often considered the worst case solution, but this section has shown that with asymmetry, and specifically with asymmetric external effects, strategic considerations by agents may lead to an even worse scenario.

\section{Conclusion}

Many natural resources are exploited in common property settings, where a fixed number of users has access to the resource and each one's decisions affect the state of the resource for everyone. Unlike free access situations, in common property the users who are sharing the resource often have some mutual knowledge of each other. Moreover, the same users have access to the resource over time. Thus, to assume that in unregulated private management each agent will behave myopically, maximizing only current profit and ignoring resource stock dynamics and neighbours' behaviour, is particularly naive. To provide a more realistic comparison of unregulated management with the first-best optimal solution requires an explicit consideration of alternative strategies for the users that do not require them to be so extremely shortsighted. One interesting possibility is to use a dynamic game setting to analyse feedback Nash equilibria, where each player is assumed to consider the dynamics of the resource as well as other players' decision rules. Such rules depend only on the current values of the state variables, not on their histories. Groundwater is a typical common property situation, as access is usually limited to owners of overlying land. External effects are pervasive, since each user's actions diminish.quantity for everyone and may have significant impacts on quality as well.

This paper develops a dynamic model of groundwater management, considering both the stock of water quantity (as represented by pumping lift) and a stock measure of water quality. The myopic and the feedback Nash solutions are examined and compared to the optimal solution. The results obtained for the symmetric case, where there is no heterogeneity among users, are similar to those in 
previous literature, namely in concluding that with feedback behaviour strategic considerations lead agents to a solution that is somewhere in between the myopic and the optimal solutions. However, explicitly considering asymmetry between water users leads to different results. The asymmetric model used in the paper is based on a situation that is frequently encountered in agricultural areas: groundwater is used for irrigation and also tapped for public supply of urban areas. These two users get different benefits from the water, and most importantly, they have very different quality requirements. Moreover, irrigated agriculture also affects the quality of groundwater. Results for the asymmetric model indicate that the strategic considerations inherent to the feedback solution may take agents even further away from the optimum than what would be expected if common property management was myopic.

Two lines of further research seem interesting: one regards the analysis of other asymmetric games in environmental and natural resource economics to establish general conditions for the emergence of strategic considerations similar to those found in the groundwater quantity-quality game. Another relates to the analysis of specific aquifers with quantity-quality problems, in the line of many previous papers that evaluate efficiency losses from unregulated vs. optimal management, to ascertain the empirical relevance of the strategic externality. Such investigations will require numerical simulations with specific functional forms.

\section{References}

Dockner, E. J. and Long, N. V. (1993), 'International pollution control: Cooperative versus noncooperative strategies', Journal of Environmental Economics and Management 24, 13-29.

Gisser, M. and Sanchez, D. A. (1980), 'Competition versus optimal control in groundwater pumping', Water Resources Research 16, 638-642.

List, J. A. and Mason, C. F. (2001), 'Optimal institutional arrangements for transboundary pollutants in a second-best world: Evidence from a differential 
game with asymmetric players', Journal of Environmental Economics and Management 42, 277-296.

Provencher, B. and Burt, O. (1993), 'The externalities associated with the common property exploitation of groundwater', Journal of Environmental Economics and Management 24, 139-158.

Roseta-Palma, C. (2002), 'Groundwater management when water quality is endogenous', Journal of Environmental Economics and Management (44), 93105.

Rubio, S. J. and Casino, B. (2001), 'Competitive versus efficient extraction of a common property resource: The groundwater case', Joumal of Economic Dynamics and Control 25, 1117-1137.

Tsutsui, S. and Mino, K. (1990), 'Nonlinear strategies in dynamic duopolistic competition with sticky prices', Journal of Economic Theory 52, 136-161.

Xepapadeas, A. (1995), 'Managing the international commons : Resource use and pollution control', Environmental and Resource Economics 5, 375-391.

Xepapadeas, A. (1996), Managing common-access resources under production externalities, in A. Xepapadeas, ed., 'Economic Policy for the Environment and Natural Resources', Edward Elgar, pp. 137-157. 